Article

\title{
A Mild and Regioselective Ring-Opening of Aziridines with Acid Anhydride Using TBD or PS-TBD as a Catalyst
}

\author{
Satoru Matsukawa * and Yasutaka Mouri \\ Department of Science Education, Faculty of Education, Ibaraki University, Ibaraki 310-8512, Japan; \\ E-Mail: c32amg777@yahoo.co.jp \\ * Author to whom correspondence should be addressed; E-Mail: satoru.matsukawa.1@vc.ibaraki.ac.jp; \\ Tel.: +81-29-228-8234; Fax: +81-29-228-8329.
}

Academic Editor: Choon-Hong Tan

Received: 2 September 2015 / Accepted: 23 September 2015 / Published: 9 October 2015

\begin{abstract}
The ring-opening of $N$-tosylaziridines with various acid anhydrides catalyzed by 5 mol \% of 1,5,7-triazabicyclo[4,4,0]dec-5-ene (TBD) afforded the corresponding $\beta$-amino esters in excellent yields under mild reaction conditions. Polymer-supported catalyst, PS-TBD also acts as a good catalyst for this reaction. PS-TBD was easily recovered and reused with minimal loss of activity.
\end{abstract}

Keywords: organocatalyst; basecatalyst; polymercatalyst; guanidine; aziridine; amino alcohols

\section{Introduction}

Aziridines are very useful intermediates for the synthesis of numerous nitrogen-containing biologically active compounds [1-3]. Therefore, nucleophilic ring-opening of aziridines by various approaches has been widely examined and developed [4-10]. $\beta$-amino alcohols, which can be synthesized from the nucleophilic ring-opening reactions of aziridines are useful intermediates in organic synthesis [4,7]. The ring-opening of aziridines with water is the most common method to prepare free $\beta$-amino alcohols. Many examples are reported to this day [11-22]. On the other hand, selectively protected $\beta$-amino alcohols are convenient intermediate to synthesize highly functionalized products. Therefore, the ring-opening reaction of aziridines with acid or acid anhydrides as the nucleophiles have been recently studied. To date, reactions using indium rtiflate [23], tributylphosphine [24], scandium triflate [25], ammonium12-molybdophosphate [26], $\mathrm{N}$-heterocyclic carbine [27] and tetrabutyl ammonium bromide [28] as 
catalysts have been reported. To develop of a more efficient reaction, we examined the use of TBD (1,5,7-Triazabicyclo[4,4,0]dec-5-ene) as a catalyst.

TBD is known as a superbase due to the high pKa of its conjugate acid [29,30]. Many unique reactions have been reported using TBD as an organocatalyst, such as the Henry reaction [31], Wittig and Horner-Wadsworth-Emmons reaction [32], Michael reaction [33], ring-opening polymerization [34], conjugate addition to activated alkenes [35], aminolysis of esters [36,37], intramolecular aldol reaction [38], and synthesis of pyrazolines [39], etc. [40-43]. Mechanistic studies of these reactions also have been discussed [44-47]. In some cases, it is considered that TBD acts as an acid-base bifunctional catalyst. Recently, we have also reported the trifluoromethylataion of aldehydes [48], and the ring-opening of aziridines with TMSCN [49] catalyzed by TBD as an organocatalyst. Herein, we report that TBD acts as an effective organobase catalyst for the ring-opening reaction of aziridines with acid anhydride.

\section{Results and Discussion}

Initially, the ring-opening reaction of $N$-tosylaziridine 1a with acetic anhydride was examined. The reaction was carried out by adding the 1a and acetic anhydride in the presence of $5 \mathrm{~mol} \%$ of TBD in DMF at $50{ }^{\circ} \mathrm{C}$. The reaction was monitored by TLC. Hydrolytic work up with saturated $\mathrm{NH}_{4} \mathrm{Cl}$ at room temperature followed by flash column chromatography afforded the ring-opened products. The product was obtained at $78 \%$ yield in $24 \mathrm{~h}$ along with $18 \%$ of the starting material. Although the reaction was performed for $48 \mathrm{~h}$, the starting material did not disappear. Then the reaction was examined at elevated temperature $\left(80^{\circ} \mathrm{C}\right)$, and the reaction proceeded smoothly. The desired product was obtained at $94 \%$ yield in $4 \mathrm{~h}$ (Table 1, entry 2). This reaction also proceeded smoothly when 2 mol \% of TBD was used (Table 1, entry 3). Among the screened solvents, DMF has proved to be the most effective for this reaction (Table 1, entries 1 vs. 4-6). The product was obtained in lower yield when other bases, such as DBU, TMG, TTMPP and DMAP (Figure 1) were used instead of TBD (Table 1, entries 1 vs. 7-10). An approximately comparable yield was observed when MTBD was used as a catalyst (Table 1, entry 11).<smiles>C1CN=C2NCCCN2C1</smiles>

(TBD)

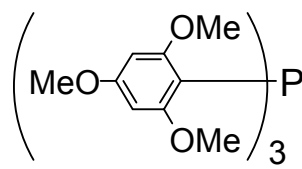

(TTMPP)<smiles>CN1CCCN2CCCN=C12</smiles>

(MTBD)<smiles>CN(C)c1ccncc1</smiles>

(DMAP)

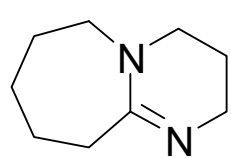

(DBU)<smiles>CN(C)C(=N)N(C)C</smiles>

(TMG)

Figure 1. Various bases. 
Table 1. Optimization of the reaction conditions.

\begin{tabular}{|c|c|c|c|}
\hline NTs & $\left.{ }_{3} \mathrm{CO}\right)_{2} \mathrm{O}$ & $\begin{array}{c}\begin{array}{c}\text { Catalyst } \\
(5 \mathrm{~mol} \%)\end{array} \\
\text { solvent } \\
80^{\circ} \mathrm{C}, 4 \mathrm{~h}\end{array}$ & $\int_{2 a}^{N H T s}$ \\
\hline Entry & Catalyst & Solvent & Yield (\%) \\
\hline 1 & TBD & $\mathrm{DMF}^{\mathrm{a}, \mathrm{b}}$ & 78 \\
\hline 2 & & DMF & 94 \\
\hline 3 & & $\mathrm{DMF}^{\mathrm{c}}$ & 90 \\
\hline 4 & & $\mathrm{THF}^{\mathrm{b}, \mathrm{d}}$ & 45 \\
\hline 5 & & $\mathrm{MeCN}^{\mathrm{b}, \mathrm{e}}$ & 10 \\
\hline 6 & & toluene ${ }^{b}$ & 71 \\
\hline 7 & DBU & $\mathrm{DMF}^{\mathrm{b}}$ & 55 \\
\hline 8 & TMG & $\mathrm{DMF}^{\mathrm{b}}$ & 33 \\
\hline 9 & DMAP & $\mathrm{DMF}^{\mathrm{b}}$ & 34 \\
\hline 10 & ТTMPР & DMF & 61 \\
\hline 11 & MTBD & $\mathrm{DMF}^{\mathrm{b}}$ & 80 \\
\hline
\end{tabular}

a at room temperature. ${ }^{\mathrm{b}}$ in $24 \mathrm{~h} .^{\mathrm{c}} 2 \mathrm{~mol} \%$ of TBD was used. ${ }^{\mathrm{d}}$ at $66^{\circ} \mathrm{C}$ (reflux condition). ${ }^{\mathrm{e}}$ at $82{ }^{\circ} \mathrm{C}$ (reflux condition).

To clarify the scope of this reaction, several $N$-tosylaziridines and acid anhydrides were examined in the presence of $5 \mathrm{~mol} \%$ TBD. In all cases, reactions were very clean and the desired products were afforded in good to excellent yields. Almost complete regioselectivity was observed when using alkyl- $N$-tosyl aziridines as substrates, and reaction on the less-substituted aziridine carbon was observed (Table 2, entries 7-10). For aryl- $N$-tosyl aziridines, in the case of a Lewis acid catalyzed reaction, selectivity demonstrated an opposite trend with alkyl- $N$-tosyl aziridines due to an electronic effect. Thus, the attack of the nucleophile at the benzylic position of aziridine occurred. However, in this Lewis base catalyzed reaction, the selectivity demonstrated the same trend: the reaction occurred on the less-substituted aziridine carbon (Table 2, entries 11-14). Reaction of propionic anhydride and benzoic anhydride also proceeded smoothly to afford the corresponding $\beta$-amino acetals in high yield. When propionic anhydride was used, regioselectivity was slightly higher than the reaction using acetic anhydride. In addition, cycloalkyl- $N$-tosyl aziridines also worked well. Unfortunately, no reaction occurred when non-acitivated aziridine such as $N$-benzylcyclohexylaziridine was employed.

Furthermore, we applied a polymer-supported TBD, PS-TBD [50-53] to this reaction. Polymer-supported catalysts have attracted significant attention in recent decades due to their inherent advantages in synthetic chemistry, e.g., simplification of reaction procedures including easy recovery of the catalyst by filtration, application to automated systems, and recycling of the catalyst [54-59]. Some unique reactions have been reported using TBD as an organocatalyst [31,60-65]. Thus, we examined the ring-opening reaction of $N$-tosylaziridine 1a with acetic anhydride in the presence of $10 \mathrm{~mol} \%$ of PS-TBD. As shown in Table 3, the reaction proceeded smoothly and the desired product was obtained at $85 \%$ yield in $10 \mathrm{~h}$ at $80{ }^{\circ} \mathrm{C}$ in DMF. A variety of $N$-tosylaziridines reacted well with acetic anhydrides to give $\beta$-amino acetates. The reaction also occurred on the less substituted aziridine carbon regardless of the type of aziridine. In addition, the recovery and reuse of PS-TBD for the reaction of 
$N$-tosylaziridine 1a with acetic anhydrides also examined. After the reaction was completed, ethyl acetate was added to the reaction mixture and the catalyst was recovered by filtration. The recovered catalyst was washed, dried and then reused. The catalyst was reused, maintaining its catalytic activity after 4 uses (Table 3, entries 1-4).

Table 2. TBD catalyzed ring-opening of various aziridines with acid anhydrides.

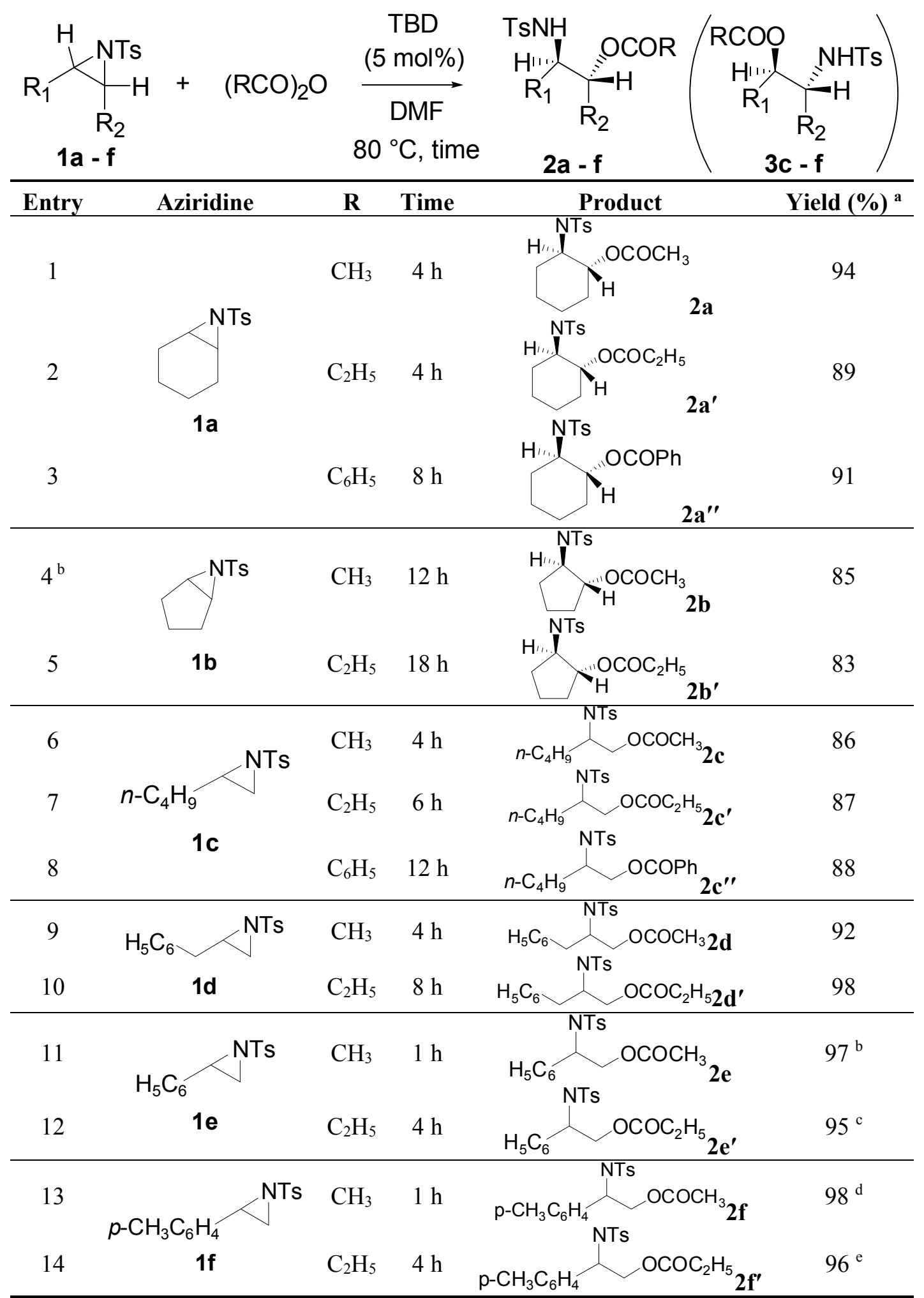

${ }^{\text {a }}$ Isolated yield. ${ }^{b}$ Regioisomer ratio $\mathbf{2 e}: \mathbf{3 e}=85: 15 .{ }^{\mathrm{c}}$ Regioisomer ratio $2 \mathbf{e}^{\prime}: \mathbf{3} \mathbf{e}^{\prime}=94: 6 .{ }^{\mathrm{d}}$ Regioisomer ratio $\mathbf{2 f : 3 f}=87: 13$. ${ }^{e}$ Regioisomer ratio $2 \mathbf{f}^{\prime}: \mathbf{3 \mathbf { f } ^ { \prime }}=90: 10$. 
Table 3. PS-TBD catalyzed ring-opening of various aziridines with acetic anhydride.<smiles>c1ccc(CN2CCCN3CCCN=C32)cc1</smiles><smiles>[R]C1C([R2])C1([R2])[S+]=C</smiles>

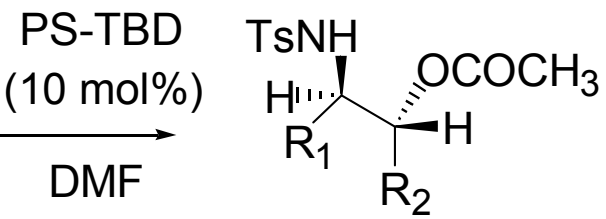

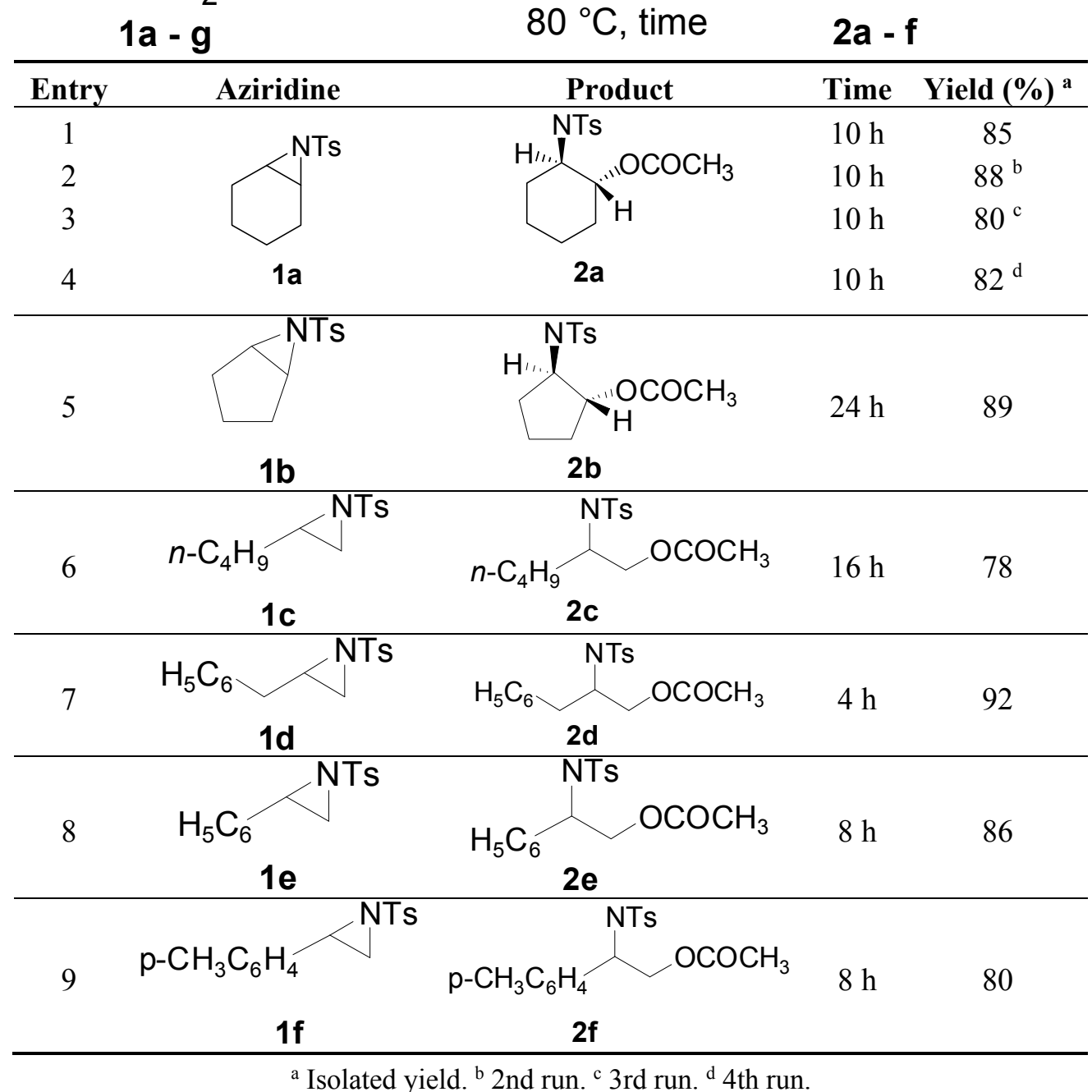

A possible mechanism is illustrated in Scheme 1. First, TBD activated the anhydride to form $\mathrm{N}^{+} \mathrm{C}(\mathrm{O}) \mathrm{R}, \mathrm{RC}(\mathrm{O}) \mathrm{O}^{-}$intermediate $\mathbf{A}$. Next, this intermediate immediately reacts with aziridines to give the ring-opening product $\mathbf{B}$. Finally, acylation occurs to give the $\mathrm{N}$-acylated adduct with regeneration of TBD.

In this transition state, a steric effect has greater priority over the electronic effect (Scheme 2). Therefore, in this Lewis base catalyzed reaction, selectivity is not dependent on a substituent. The reaction occurred on the less-substituted aziridine carbon even in the case of phenyl substituted aziridine 1e. 


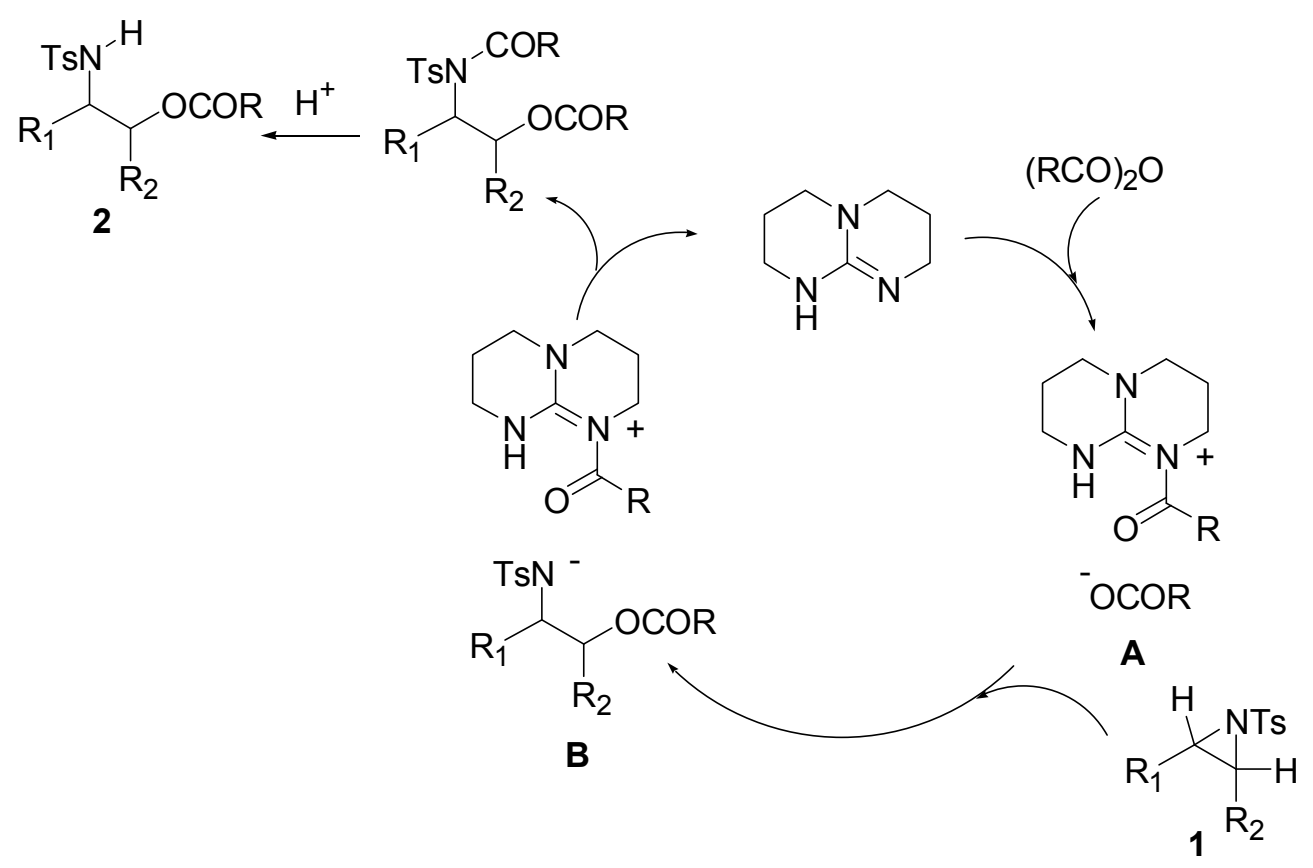

Scheme 1. Proposed mechanism.
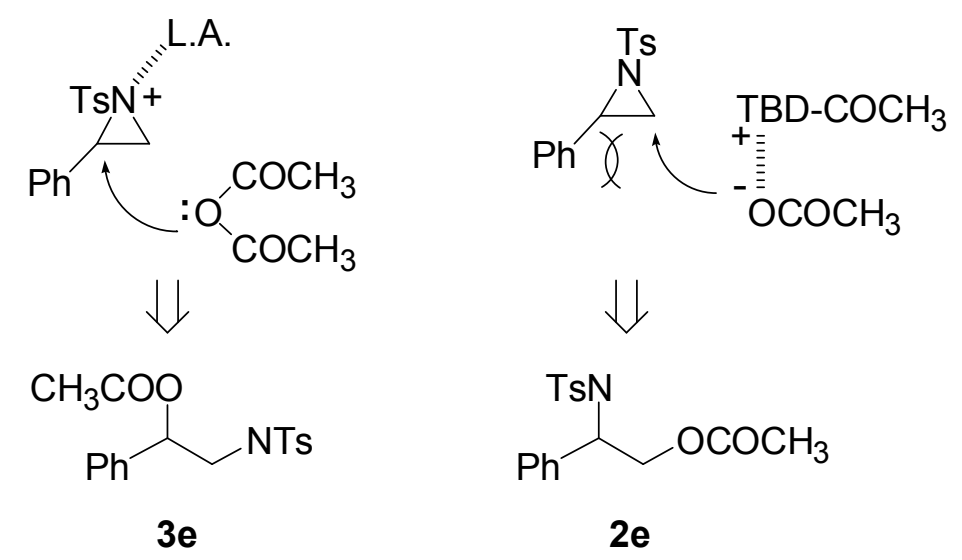

Scheme 2. The origin of regioselectivity.

\section{Experimental Section}

\subsection{General}

All reactions were performed under an argon atmosphere using oven-dried glassware. Flash column chromatography was performed using silica gel Wakogel C-200 (Wako Chemical, Osaka, Japan). Preparative thin-layer chromatography was carried out on silica gel Wakogel B-5F (Wako Chemical). Dehydrate DMF, THF, toluene and $\mathrm{CH}_{3} \mathrm{CN}$ were purchased from Wako Chemical. Other commercially available reagent was used as received without further purification. The aziridines were prepared according to literature procedure [66]. Yields refer to isolated compounds estimated to be $>95 \%$ pure, as determined by ${ }^{1} \mathrm{H}-\mathrm{NMR}$ spectroscopy. IR spectra were recorded on a JUSCO FT/IR-430 spectrometer (JASCO Corporation, Tokyo, Japan). ${ }^{1} \mathrm{H}$ - and ${ }^{13} \mathrm{C}-\mathrm{NMR}$ spectra were determined for solutions in $\mathrm{CDCl}_{3}$ with $\mathrm{Me}_{4} \mathrm{Si}$ as internal standard on a Bruker Avance III instrument (Bruker Corporation, Billerica, MA, USA). HRMS data were measured on a JEOL JMS-700 mass spectrometer (JEOL Ltd., Tokyo, Japan). 


\subsection{Method}

\subsubsection{General Procedure for TBD-Catalyzed Ring-Opening of Aziridines with Acid Anhydride}

To a solution of TBD $(0.05 \mathrm{mmol})$ in DMF $(1 \mathrm{~mL})$ was added aziridine $(1.0 \mathrm{mmol})$ and acid anhydride (1.25 mmol) at room temperature. After the reaction was complete (as determinedby TLC), the reaction mixture was washed with saturated $\mathrm{NH}_{4} \mathrm{Cl}$ and extracted with EtOAc $(2 \times 10 \mathrm{~mL})$. The combined organic layers were dried over $\mathrm{Na}_{2} \mathrm{SO}_{4}$, concentrated in vacuo and purified by column chromatography on silica gel (EtOAc:hexane $=1: 3)$ to give the corresponding product.

\subsubsection{General Procedure for PS-TBD Catalyzed Ring-Opening of Aziridines with Acid Anhydride}

To a solution of PS-TBD $(0.10 \mathrm{mmol})$ in DMF $(1 \mathrm{~mL})$ was added aziridine $(1.0 \mathrm{mmol})$ and acid anhydride (1.25 mmol) at room temperature. After the reaction was complete (as determined by TLC), EtOAc $(5 \mathrm{~mL})$ was added to the mixture and PS-TBD was separated by filtration. The filtrate was washed with saturated $\mathrm{NH}_{4} \mathrm{Cl}$, dried over $\mathrm{Na}_{2} \mathrm{SO}_{4}$. The organic layer was concentrated in vacuo and purified by column chromatography on silica gel (EtOAc:hexane $=1: 3$ ) to give the corresponding product. The recovered catalyst is reusable after washing (acetone and water) and drying in vacuo.

\subsection{General Characterization of the Products}

2-(4-Methylphenylsulfonamido)cyclohexyl acetate (2a) [23]. Colorless plate; m.p. $108-110{ }^{\circ} \mathrm{C}$; yield: $294 \mathrm{mg}$ (94\%); IR (KBr): 3030, 2929, 2195, 1644, 1598, 1449, 823, $700 \mathrm{~cm}^{-1}$; ${ }^{1} \mathrm{H}-\mathrm{NMR}(500 \mathrm{~Hz}$, $\left.\mathrm{CDCl}_{3}\right) \delta 1.16-1.32(\mathrm{~m}, 4 \mathrm{H}), 1.57-1.61(\mathrm{~m}, 1 \mathrm{H}), 1.62-1.66(\mathrm{~m}, 1 \mathrm{H}), 1.74(\mathrm{~s}, 3 \mathrm{H}), 1.85-1.89(\mathrm{~m}, 1 \mathrm{H})$, $1.96-2.00(\mathrm{~m}, 1 \mathrm{H}), 2.38(\mathrm{~s}, 3 \mathrm{H}), 3.12-3.18(\mathrm{~m}, 1 \mathrm{H}), 4.53(\mathrm{dt}, J=4.6,10.4 \mathrm{~Hz}, 1 \mathrm{H}), 4.95(\mathrm{~d}, J=6.8 \mathrm{~Hz}$, $1 \mathrm{H}), 7.25(\mathrm{~d}, J=8.0 \mathrm{~Hz}, 2 \mathrm{H}), 7.71(\mathrm{~d}, J=8.0 \mathrm{~Hz}, 2 \mathrm{H}) ;{ }^{13} \mathrm{C}-\mathrm{NMR}\left(125 \mathrm{MHz}, \mathrm{CDCl}_{3}\right) \delta 20.9,21.5$, 23.6, 24.2, 31.1, 33.5, 56.9, 74.1, 126.9, 129.6, 138.7, 143.0, 171.4; HRMS (FAB): $m / z$ : cald for $\mathrm{C}_{15} \mathrm{H}_{22} \mathrm{NO}_{4} \mathrm{~S}: 312.1270$; found: $312.1280[\mathrm{M}+\mathrm{H}]^{+}$.

2-(4-Methylphenylsulfonamido) cyclohexyl propionate (2a') [23]. Colorless cube; m.p. 92-94 ${ }^{\circ} \mathrm{C}$; yield: $289 \mathrm{mg}(89 \%)$; IR (KBr): 3284, 2957, 1736, 1460, 1331, 1162, 1092, 815, $666 \mathrm{~cm}^{-1}$; ${ }^{1} \mathrm{H}-\mathrm{NMR}$ $\left(500 \mathrm{~Hz}, \mathrm{CDCl}_{3}\right) \delta 1.02(\mathrm{t}, J=7.5 \mathrm{~Hz}, 3 \mathrm{H}), 1.20-1.39(\mathrm{~m}, 4 \mathrm{H}), 1.62-1.67(\mathrm{~m}, 1 \mathrm{H}), 1.68-1.71(\mathrm{~m}, 1 \mathrm{H})$, 1.95-1.99 (m, 1H), 2.00-2.05 (m, 1H), 2.07 (q, $J=7.5 \mathrm{~Hz}, 2 \mathrm{H}), 2.42(\mathrm{~s}, 3 \mathrm{H}), 3.19-3.24(\mathrm{~m}, 1 \mathrm{H}), 4.59$ (dq, $J=3.4,10.4 \mathrm{~Hz}, 1 \mathrm{H}), 5.04(\mathrm{~d}, J=10.4 \mathrm{~Hz}, 1 \mathrm{H}), 7.29$ (d, $J=8.6 \mathrm{~Hz}, 2 \mathrm{H}), 7.74(\mathrm{~d}, J=8.6 \mathrm{~Hz}, 2 \mathrm{H})$; ${ }^{13} \mathrm{C}-\mathrm{NMR}\left(125 \mathrm{MHz}, \mathrm{CDCl}_{3}\right) \delta$ 8.8, 21.4, 23.6, 24.1, 27.4, 31.0, 33.3, 56.9, 73.8, 126.8, 129.5, 138.7, 142.9, 174.8; HRMS (FAB): $m / z$ : cald for $\mathrm{C}_{16} \mathrm{H}_{24} \mathrm{NO}_{4} \mathrm{~S}$ : 326.1426 ; found: $326.1410[\mathrm{M}+\mathrm{H}]^{+}$.

2-(4-Methylphenylsulfonamido)-1-phenylcarbonyloxyclohexane (2a") [24]. White solid; m.p. 128-130 ${ }^{\circ} \mathrm{C}$; yield: $340 \mathrm{mg}$ (91\%); IR (KBr): 3321, 2926, 1786, 1702, 1598, 1450, 1320, 1212, 1155, 1014, 715, $666 \mathrm{~cm}^{-1} ;{ }^{1} \mathrm{H}-\mathrm{NMR}\left(500 \mathrm{~Hz}, \mathrm{CDCl}_{3}\right) \delta 1.20-1.28(\mathrm{~m}, 2 \mathrm{H}), 1.27-1.42(\mathrm{~m}, 2 \mathrm{H}), 1.64-1.66(\mathrm{~m}, 1 \mathrm{H})$, $1.67-1.71(\mathrm{~m}, 1 \mathrm{H}), 1.95-2.01(\mathrm{~m}, 1 \mathrm{H}), 2.10-2.17(\mathrm{~m}, 1 \mathrm{H}), 2.15(\mathrm{~s}, 3 \mathrm{H}), 3.26-3.32(\mathrm{~m}, 1 \mathrm{H}), 4.79(\mathrm{dt}$, $J=4.7,10.7 \mathrm{~Hz}, 1 \mathrm{H}), 5.16(\mathrm{~d}, J=7.4 \mathrm{~Hz}, 1 \mathrm{H}), 6.88(\mathrm{~d}, J=8.0 \mathrm{~Hz}, 2 \mathrm{H}), 7.32(\mathrm{~d}, J=8.0 \mathrm{~Hz}, 2 \mathrm{H}), 7.50$ $(\mathrm{d}, J=6.4 \mathrm{~Hz}, 1 \mathrm{H}), 7.55(\mathrm{~d}, J=8.2 \mathrm{~Hz}, 2 \mathrm{H}), 7.74(\mathrm{~d}, J=7.0 \mathrm{~Hz}, 2 \mathrm{H}) ;{ }^{13} \mathrm{C}-\mathrm{NMR}\left(125 \mathrm{MHz}, \mathrm{CDCl}_{3}\right) \delta$ 
21.2, 23.6, 24.1, 31.1, 33.8, 57.1, 74.5, 126.4, 128.0, 128.8, 129.3, 129.6, 130.4, 132.8, 138.0, 142.5, 166.6; HRMS (FAB): $m / z$ : cald for $\mathrm{C}_{20} \mathrm{H}_{24} \mathrm{NO}_{4} \mathrm{~S}$ : 374.1426 ; found: $374.1420[\mathrm{M}+\mathrm{H}]^{+}$.

2-(4-Methylphenylsulfonamido)cyclopentyl acetate (2b) [23]. Colorless oil; yield: $253 \mathrm{mg}$ (85\%); IR (neat): 3278, 2925, 1732, 1455, 1326, 1158, 1092, 815, $666 \mathrm{~cm}^{-1} ;{ }^{1} \mathrm{H}-\mathrm{NMR}\left(500 \mathrm{~Hz}, \mathrm{CDCl}_{3}\right) \delta$ 1.44-1.50 (m, 2H), 1.57-1.64 (m, 2H), $1.84(\mathrm{~s}, 3 \mathrm{H}), 1.95-1.99(\mathrm{~m}, 2 \mathrm{H}), 2.39(\mathrm{~s}, 3 \mathrm{H}), 3.40-3.44(\mathrm{~m}, 1 \mathrm{H})$, $4.82(\mathrm{dt}, J=5.5,7.5 \mathrm{~Hz}, 1 \mathrm{H}), 5.33-5.39$ (brs, $1 \mathrm{H}), 7.26(\mathrm{~d}, J=8.1 \mathrm{~Hz}, 2 \mathrm{H}), 7.72(\mathrm{~d}, J=8.1 \mathrm{~Hz}, 2 \mathrm{H})$; ${ }^{13} \mathrm{C}-\mathrm{NMR}\left(125 \mathrm{MHz}, \mathrm{CDCl}_{3}\right) \delta$ 20.7, 20.8, 21.4, 29.3, 31.0, 59.7, 79.5, 127.1, 129.5, 137.3, 143.3, 171.0; HRMS (FAB): $m / z$ : cald for $\mathrm{C}_{14} \mathrm{H}_{20} \mathrm{NO}_{4} \mathrm{~S}$ : 298.1113 ; found: $298.1118[\mathrm{M}+\mathrm{H}]^{+}$.

2-(4-Methylphenylsulfonamido)cyclopentyl propionate (2b') [25]. Colorless oil; yield: $258 \mathrm{mg}$ (83\%); IR (neat): 3276, 2941, 1741, 1462, 1356, 1165, 813, $666 \mathrm{~cm}^{-1} ;{ }^{1} \mathrm{H}-\mathrm{NMR}\left(500 \mathrm{~Hz}, \mathrm{CDCl}_{3}\right) \delta 0.94$ (t, $J=7.6 \mathrm{~Hz}, 3 \mathrm{H}), 1.40-1.45(\mathrm{~m}, 2 \mathrm{H}), 1.50-1.57(\mathrm{~m}, 2 \mathrm{H}), 1.85-1.95(\mathrm{~m}, 2 \mathrm{H}), 2.00-2.10(\mathrm{~m}, 2 \mathrm{H}), 2.32$ (s, 3H), 3.33-3.40 (m, 1H), $4.80(\mathrm{dt}, J=5.4,7.4 \mathrm{~Hz}, 1 \mathrm{H}), 5.70(\mathrm{~d}, J=7.4 \mathrm{~Hz}, 1 \mathrm{H}), 7.21(\mathrm{~d}, J=8.1 \mathrm{~Hz}$, 2H), $7.68(\mathrm{~d}, J=8.1 \mathrm{~Hz}, 2 \mathrm{H}) ;{ }^{13} \mathrm{C}-\mathrm{NMR}\left(125 \mathrm{MHz}, \mathrm{CDCl}_{3}\right) \delta 8.7,20.6,27.2,29.2,30.8,59.4,79.2$, 126.9, 129.3, 137.3, 143.1, 174.2; HRMS (FAB): $m / z$ : cald for $\mathrm{C}_{15} \mathrm{H}_{22} \mathrm{NO}_{4} \mathrm{~S}$ : 312.1270 ; found: $312.1285[\mathrm{M}+\mathrm{H}]^{+}$.

2-(4-Methylphenylsulfonamido)hexyl acetate (2c) [23]. Colorless oil; yield: $269 \mathrm{mg}$ (86\%); IR (neat): 3281, 2957, 2871, 1741, 1598, 1431, 1329, 1239, 1162, 1093, 815, $666 \mathrm{~cm}^{-1}$; ${ }^{1} \mathrm{H}-\mathrm{NMR}(500 \mathrm{~Hz}$, $\left.\mathrm{CDCl}_{3}\right) \delta 0.76(\mathrm{t}, J=7.2 \mathrm{~Hz}, 3 \mathrm{H}), 1.06-1.30(\mathrm{~m}, 4 \mathrm{H}), 1.32-1.45(\mathrm{~m}, 2 \mathrm{H}), 1.90(\mathrm{~s}, 3 \mathrm{H}), 2.39(\mathrm{~s}, 3 \mathrm{H})$, $3.38-3.43(\mathrm{~m}, 1 \mathrm{H}), 3.85(\mathrm{dd}, J=4.3,11.4 \mathrm{~Hz}, 1 \mathrm{H}), 3.94(\mathrm{dd}, J=5.4,11.4 \mathrm{~Hz}, 1 \mathrm{H}), 4.90(\mathrm{~d}, J=8.5 \mathrm{~Hz}$, $1 \mathrm{H}), 7.26(\mathrm{~d}, J=7.9 \mathrm{~Hz}, 2 \mathrm{H}), 7.73(\mathrm{~d}, J=7.9 \mathrm{~Hz}, 2 \mathrm{H}) ;{ }^{13} \mathrm{C}-\mathrm{NMR}\left(125 \mathrm{MHz}, \mathrm{CDCl}_{3}\right) \delta 13.7,20.6$, 21.4, 22.2, 27.4, 32.0, 52.8, 65.7, 127.0, 129.6, 138.1, 143.3, 170.8; HRMS (FAB): $m / z$ : cald for $\mathrm{C}_{15} \mathrm{H}_{24} \mathrm{NO}_{4} \mathrm{~S}: 314.1426$; found: $314.1409[\mathrm{M}+\mathrm{H}]^{+}$.

2-(4-Methylphenylsulfonamido)hexyl propionate (2c') [26]. Colorless oil; yield: $285 \mathrm{mg}$ (87\%); IR (neat): 3283, 2957, 1740, 1462, 1330, 1162, 1092, 815, $667 \mathrm{~cm}^{-1} ;{ }^{1} \mathrm{H}-\mathrm{NMR}\left(500 \mathrm{~Hz}, \mathrm{CDCl}_{3}\right) \delta 0.76$ (t, $J=7.1 \mathrm{~Hz}, 3 \mathrm{H}), 1.05(\mathrm{t}, J=7.6 \mathrm{~Hz}, 3 \mathrm{H}), 1.15-1.25(\mathrm{~m}, 4 \mathrm{H}), 1.35-1.48(\mathrm{~m}, 2 \mathrm{H}), 2.18$ (q, $J=7.6 \mathrm{~Hz}$, $2 \mathrm{H}), 2.39(\mathrm{~s}, 3 \mathrm{H}), 3.36-3.45(\mathrm{~m}, 1 \mathrm{H}), 3.86(\mathrm{dd}, J=4.3,11.4 \mathrm{~Hz}, 1 \mathrm{H}), 3.96(\mathrm{dd}, J=5.5,11.4 \mathrm{~Hz}, 1 \mathrm{H})$, $4.77-4.82(\mathrm{~m}, 1 \mathrm{H}), 7.26(\mathrm{~d}, J=8.4 \mathrm{~Hz}, 2 \mathrm{H}), 7.73(\mathrm{~d}, J=8.4 \mathrm{~Hz}, 2 \mathrm{H}) ;{ }^{13} \mathrm{C}-\mathrm{NMR}\left(125 \mathrm{MHz}, \mathrm{CDCl}_{3}\right) \delta$ 8.9, 13.7, 21.4, 22.2, 27.1, 27.4, 32.0, 52.9, 65.6, 126.9, 129.6, 138.1, 143.3, 174.2; HRMS (FAB): $m / z$ : cald for $\mathrm{C}_{16} \mathrm{H}_{26} \mathrm{NO}_{4} \mathrm{~S}: 328.1583$; found: $328.1596[\mathrm{M}+\mathrm{H}]^{+}$.

2-(4-Methylphenylsulfonamido)-1-phenylcarbonyloxyhexane (2c'") [23]. White solid; m.p. 65-69 ${ }^{\circ} \mathrm{C}$; yield: $330 \mathrm{mg}(88 \%)$; IR (KBr): 3288, 2929, 1787, 1560, 1452, 1273, 1162, 815, 708, $615 \mathrm{~cm}^{-1}$; ${ }^{1} \mathrm{H}-\mathrm{NMR}\left(500 \mathrm{~Hz}, \mathrm{CDCl}_{3}\right) \delta 0.78(\mathrm{t}, J=7.2 \mathrm{~Hz}, 3 \mathrm{H}), 1.13-1.31(\mathrm{~m}, 4 \mathrm{H}), 1.45-1.59(\mathrm{~m}, 2 \mathrm{H}), 2.29(\mathrm{~s}, 3 \mathrm{H})$, $3.53-3.62(\mathrm{~m}, 1 \mathrm{H}), 4.11(\mathrm{dd}, J=4.2,11.5 \mathrm{~Hz}, 1 \mathrm{H}), 4.22(\mathrm{dd}, J=5.6,11.5 \mathrm{~Hz}, 1 \mathrm{H}), 5.16(\mathrm{~d}, J=8.3 \mathrm{~Hz}$, $1 \mathrm{H}), 7.11(\mathrm{~d}, J=8.0 \mathrm{~Hz}, 2 \mathrm{H}), 7.36(\mathrm{t}, J=7.5 \mathrm{~Hz}, 2 \mathrm{H}), 7.52(\mathrm{t}, J=7.5 \mathrm{~Hz}, 1 \mathrm{H}), 7.71(\mathrm{~d}, J=8.3 \mathrm{~Hz}$, 2H), $7.89(\mathrm{~d}, J=7.1 \mathrm{~Hz}, 2 \mathrm{H}) ;{ }^{13} \mathrm{C}-\mathrm{NMR}\left(125 \mathrm{MHz}, \mathrm{CDCl}_{3}\right) \delta 13.7,21.4,22.2,27.5,32.2,53.0,66.2$, 126.8, 128.2, 129.6, 133.1, 138.0, 143.2, 166.2; HRMS (FAB): $m / z$ : cald for $\mathrm{C}_{20} \mathrm{H}_{26} \mathrm{NO}_{4} \mathrm{~S}: 376.1583$; found: $374.1564[\mathrm{M}+\mathrm{H}]^{+}$. 
2-(4-Methylphenylsulfonamido)-3-phenylpropyl acetate (2d) [23]. White solid; m.p. 67-69 ${ }^{\circ} \mathrm{C}$; yield: $320 \mathrm{mg}$ (92\%); IR (KBr): 3280, 2953, 1742, 1455, 1329, 1160, 1092, 815, $667 \mathrm{~cm}^{-1}$; ${ }^{1} \mathrm{H}-\mathrm{NMR}(500 \mathrm{~Hz}$, $\left.\mathrm{CDCl}_{3}\right) \delta 1.93(\mathrm{~s}, 3 \mathrm{H}), 2.37(\mathrm{~s}, 3 \mathrm{H}), 2.75(\mathrm{~d}, J=7.0 \mathrm{~Hz}, 2 \mathrm{H}), 3.62-3.69(\mathrm{~m}, 1 \mathrm{H}), 3.90(\mathrm{dd}, J=5.3,15.0 \mathrm{~Hz}$, $1 \mathrm{H}), 3.92(\mathrm{dd}, J=5.4,15.0 \mathrm{~Hz}, 1 \mathrm{H}), 5.18(\mathrm{~d}, J=8.1 \mathrm{~Hz}, 1 \mathrm{H}), 7.00$ (d, $J=8.0 \mathrm{~Hz}, 2 \mathrm{H}), 7.14-7.22$ (m, $5 \mathrm{H}), 7.62(\mathrm{~d}, J=8.2 \mathrm{~Hz}, 2 \mathrm{H}) ;{ }^{13} \mathrm{C}-\mathrm{NMR}\left(125 \mathrm{MHz}, \mathrm{CDCl}_{3}\right) \delta 20.6,21.3,38.5,53.8,64.8,126.7,126.8$, 128.6, 129.1, 129.5, 136.2, 137.5, 143.2, 170.7; HRMS (FAB): $m / z$ : cald for $\mathrm{C}_{18} \mathrm{H}_{22} \mathrm{NO}_{4} \mathrm{~S}: 348.1270$; found: $348.1254[\mathrm{M}+\mathrm{H}]^{+}$.

2-(4-Methylphenylsulfonamido)-3-phenylpropyl propionate (2d') [25]. Colorless oil; yield: $354 \mathrm{mg}$ (98\%); IR (neat): 3281, 2942, 1740, 1456, 1330, 1160, 1092, 814, $666 \mathrm{~cm}^{-1} ;{ }^{1} \mathrm{H}-\mathrm{NMR}\left(500 \mathrm{~Hz}, \mathrm{CDCl}_{3}\right)$ $\delta 1.08(\mathrm{t}, J=7.6 \mathrm{~Hz}, 3 \mathrm{H}), 2.20(\mathrm{q}, J=7.6 \mathrm{~Hz}, 2 \mathrm{H}), 2.40(\mathrm{~s}, 3 \mathrm{H}), 3.34-3.41(\mathrm{~m}, 1 \mathrm{H}), 3.92(\mathrm{dd}, J=4.7$, $11.5 \mathrm{~Hz}, 1 \mathrm{H}), 3.97(\mathrm{dd}, J=5.5,11.5 \mathrm{~Hz}, 1 \mathrm{H}), 4.84(\mathrm{~d}, J=7.9 \mathrm{~Hz}, 1 \mathrm{H}), 7.02(\mathrm{~d}, J=7.3 \mathrm{~Hz}, 2 \mathrm{H}), 7.16-7.23$ (m, 5H), $7.63(\mathrm{~d}, J=8.3 \mathrm{~Hz}, 2 \mathrm{H}) ;{ }^{13} \mathrm{C}-\mathrm{NMR}\left(125 \mathrm{MHz}, \mathrm{CDCl}_{3}\right) \delta 8.9,21.4,27.2,38.5,53.9,64.7,126.8$, $126.9,128.7,129.2,129.6,136.2,137.6,143.3,174.1$; HRMS (FAB): $m / z$ : cald for $\mathrm{C}_{19} \mathrm{H}_{24} \mathrm{NO}_{4} \mathrm{~S}$ : 362.1426; found: $362.1445[\mathrm{M}+\mathrm{H}]^{+}$.

2-(4-Methylphenylsulfonamido)-2-phenylethyl acetate (2e) [23]. Colorless oil; yield: $300 \mathrm{mg}$ (90\%); IR (neat): 3280, 2923, 1742, 1434, 1328, 1160, 1092, 814, $666 \mathrm{~cm}^{-1} ;{ }^{1} \mathrm{H}-\mathrm{NMR}\left(500 \mathrm{~Hz}, \mathrm{CDCl}_{3}\right) \delta 1.91$ (s, 3H), $2.35(\mathrm{~s}, 3 \mathrm{H}), 4.14(\mathrm{dd}, J=4.9,11.6 \mathrm{~Hz}, 1 \mathrm{H}), 4.18(\mathrm{dd}, J=7.9,11.6 \mathrm{~Hz}, 1 \mathrm{H}), 4.60(\mathrm{dt}, J=4.9$, $7.3 \mathrm{~Hz}, 1 \mathrm{H}), 5.43(\mathrm{~d}, J=7.0 \mathrm{~Hz}, 1 \mathrm{H}), 7.08-7.12(\mathrm{~m}, 4 \mathrm{H}), 7.16-7.18(\mathrm{~m}, 2 \mathrm{H}), 7.26-7.30(\mathrm{~m}, 1 \mathrm{H}), 7.57$ $(\mathrm{d}, J=8.4 \mathrm{~Hz}, 2 \mathrm{H}) ;{ }^{13} \mathrm{C}-\mathrm{NMR}\left(125 \mathrm{MHz}, \mathrm{CDCl}_{3}\right) \delta 20.6,21.3,56.9,66.5,126.8,127.1,128.6,129.4$, 137.0, 137.5, 143.3, 170.8; HRMS (FAB): $m / z$ : cald for $\mathrm{C}_{17} \mathrm{H}_{20} \mathrm{NO}_{4} \mathrm{~S}: 334.1113$; found: 334.1119 [M+ H] $]^{+}$

2-(4-Methylphenylsulfonamido)-2-phenylethyl propionate (2e') [25]. Colorless oil; yield: $306 \mathrm{mg}$ (88\%); IR (neat): 3280, 2924, 1741, 1516, 1438, 1160, 1090, 813, $666 \mathrm{~cm}^{-1} ;{ }^{1} \mathrm{H}-\mathrm{NMR}\left(500 \mathrm{~Hz}, \mathrm{CDCl}_{3}\right) \delta 1.02$ (t, $J=7.6 \mathrm{~Hz}, 3 \mathrm{H}), 2.18$ (q, $J=7.6 \mathrm{~Hz}, 2 \mathrm{H}), 2.35$ (s, 3H), 4.17 (dd, $J=5.0 \mathrm{~Hz}, 11.6 \mathrm{~Hz}, 1 \mathrm{H}), 4.20$ (dd, $J=7.4,11.6 \mathrm{~Hz}, 1 \mathrm{H}), 4.62(\mathrm{dt}, J=4.9,7.3 \mathrm{~Hz}, 1 \mathrm{H}), 5.72(\mathrm{~d}, 6.6 \mathrm{~Hz}, 1 \mathrm{H}), 7.12-7.15(\mathrm{~m}, 4 \mathrm{H}), 7.17-7.19$ $(\mathrm{m}, 2 \mathrm{H}), 7.27-7.30(\mathrm{~m}, 1 \mathrm{H}), 7.59(\mathrm{~d}, J=8.3 \mathrm{~Hz}, 2 \mathrm{H}) ;{ }^{13} \mathrm{C}-\mathrm{NMR}\left(125 \mathrm{MHz}, \mathrm{CDCl}_{3}\right) \delta 8.8,21.3,27.1$, 56.9, 66.3, 126.8, 127.0, 128.5, 129.3, 137.1, 137.4, 143.1, 174.2; HRMS (FAB): $m / z$ : cald for $\mathrm{C}_{18} \mathrm{H}_{22} \mathrm{NO}_{4} \mathrm{~S}: 348.1270$; found: $348.1262[\mathrm{M}+\mathrm{H}]^{+}$.

2-(4-Methylphenylsulfonamido)-2-(4-methylphenyl)ethyl acetate (2f) [23]. White solid; m.p. 88-90 ${ }^{\circ} \mathrm{C}$; yield: $316 \mathrm{mg}$ (91\%); IR (neat): 3278, 2925, 1742, 1495, 1330, 1161, 1044, 815, $668 \mathrm{~cm}^{-1}$; ${ }^{1} \mathrm{H}-\mathrm{NMR}$ $\left(500 \mathrm{~Hz}, \mathrm{CDCl}_{3}\right) \delta 1.93(\mathrm{~s}, 3 \mathrm{H}), 2.28(\mathrm{~s}, 3 \mathrm{H}), 2.38(\mathrm{~s}, 3 \mathrm{H}), 4.14(\mathrm{dd}, J=4.8,11.6 \mathrm{~Hz}, 1 \mathrm{H}), 4.19$ $(\mathrm{dd}, J=7.6,11.6 \mathrm{~Hz}, 1 \mathrm{H}), 4.56(\mathrm{dt}, J=4.9,7.3 \mathrm{~Hz}, 1 \mathrm{H}), 5.66(\mathrm{dd}, J=5.0,7.3 \mathrm{~Hz}, 1 \mathrm{H}), 7.00-7.02$ $(\mathrm{m}, 4 \mathrm{H}), 7.17(\mathrm{~d}, J=8.0 \mathrm{~Hz}, 2 \mathrm{H}), 7.60(\mathrm{~d}, J=8.0 \mathrm{~Hz}, 2 \mathrm{H}) ;{ }^{13} \mathrm{C}-\mathrm{NMR}\left(125 \mathrm{MHz}, \mathrm{CDCl}_{3}\right) \delta 20.6,21.0$, 21.4, 56.5, 66.4, 126.7, 127.0, 129.2, 129.3, 133.9, 137.4, 137.8, 143.2, 170.8; HRMS (FAB): $m / z$ : cald for $\mathrm{C}_{18} \mathrm{H}_{22} \mathrm{NO}_{4} \mathrm{~S}: 348.1270$; found: $348.1288[\mathrm{M}+\mathrm{H}]^{+}$.

2-(4-Methylphenylsulfonamido)-2-(4-methylphenyl)ethyl acetate $\left(\mathbf{2 f}^{\prime}\right)$ [25]. White solid; m.p. 98-100 ${ }^{\circ} \mathrm{C}$; yield: $310 \mathrm{mg}$ (83\%); IR (neat): 3283, 2982, 1740, 1598, 1462, 1348, 1161, 1088, 814, $666 \mathrm{~cm}^{-1}$; ${ }^{1} \mathrm{H}-\mathrm{NMR}\left(500 \mathrm{~Hz}, \mathrm{CDCl}_{3}\right) \delta 1.04(\mathrm{t}, J=7.6 \mathrm{~Hz}, 3 \mathrm{H}), 2.20(\mathrm{q}, J=7.6 \mathrm{~Hz}, 2 \mathrm{H}), 2.28(\mathrm{~s}, 3 \mathrm{H}), 2.38$ (s, 3H), $4.15(\mathrm{dd}, J=4.8,11.6 \mathrm{~Hz}, 1 \mathrm{H}), 4.20(\mathrm{dd}, J=7.6,11.6 \mathrm{~Hz}, 1 \mathrm{H}), 4.57(\mathrm{dt}, J=4.8,7.2 \mathrm{~Hz}, 1 \mathrm{H})$, 
$5.22(\mathrm{~d}, J=6.8 \mathrm{~Hz}, 1 \mathrm{H}), 7.00-7.02(\mathrm{~m}, 4 \mathrm{H}), 7.17(\mathrm{~d}, J=8.0 \mathrm{~Hz}, 2 \mathrm{H}), 7.59(\mathrm{~d}, J=8.0 \mathrm{~Hz}, 2 \mathrm{H})$; ${ }^{13} \mathrm{C}-\mathrm{NMR}\left(125 \mathrm{MHz}, \mathrm{CDCl}_{3}\right) \delta 8.8,21.0,21.4,56.7,66.3,126.7,127.0,129.2,129.4,134.0,137.4$, 137.9, 143.2, 174.3; HRMS (FAB): $m / z$ : cald for $\mathrm{C}_{19} \mathrm{H}_{24} \mathrm{NO}_{4} \mathrm{~S}: 362.1426$; found: 362.1415 [M $\left.+\mathrm{H}\right]^{+}$.

Copies of ${ }^{1} \mathrm{H}$ - and ${ }^{13} \mathrm{C}-\mathrm{NMR}$ Spectra of products $\mathbf{2} \mathbf{a}-\mathbf{2} \mathbf{f}, \mathbf{2} \mathbf{a}^{\prime}-\mathbf{2} \mathbf{f}^{\prime}, \mathbf{2} \mathbf{a}^{\prime \prime}$, and $\mathbf{2} \mathbf{c}^{\prime \prime}$ could be found in the supplementary materials.

\section{Conclusions}

In conclusion, we have demonstrated TBD catalyzed ring-opening reactions of $N$-tosylaziridine with acid anhydrides. A broad range of $N$-tosylaziridine and acid anhydrides could be applied using 5 mol \% TBD. Furthermore, polymer-supported catalyst, PS-TBD also act as a good catalyst for this reaction. PS-TBD was easily recovered and reused with minimal loss of activity. These reactions provide a simple and convenient method for the synthesis of highly functionalized $\beta$-amino alcohols.

\section{Supplementary Materials}

Supplementary materials can be accessed at: http://www.mdpi.com/1420-3049/20/10/18482/s1.

\section{Author Contributions}

S.M. conceived the ideas, analyzed the data, and wrote the paper; Y.M. performed the experiments, analyzed the data; All authors read and approved the final manuscript.

\section{Conflicts of Interest}

The authors declare no conflict of interest.

\section{References}

1. Sweeney, J.B. Aziridines: Epoxides' ugly cousins? Chem. Soc. Rev. 2002, 31, 247-258.

2. Padwa, A. Pearson, W.H.; Lian, B.N.; Bergmeier, S.C. In Comprehensive Heterocyclic Chemistry II; Katritzky, A.R., Rees, C.W., Scriven, E.F.V., Eds.; Pergamon: New York, NY, USA, 1996; Volume 1a, pp. 1-96.

3. Tanner, D. Chiral Aziridines-Their Synthesis and Use in Stereoselective Transformations. Angew. Chem. Int. Ed. Engl. 1994, 33, 599-619.

4. $\mathrm{Hu}, \mathrm{X}$.E. Nucleophilic ring opening of aziridines. Tetrahedron 2004, 60, 2701-2743.

5. Watson, I.D.G.; Yu, L.; Yudin, A.K. Advances in nitrogen transfer reactions involving aziridines. Acc. Chem. Res. 2006, 39, 194-206.

6. Singh, G.S.; D'hooghe, M.; Kimpe, N.D. Aziridines synthesis and reactivity of C-heteroatomsubstituted aziridines. Chem. Rev. 2007, 107, 2080-2135.

7. Lu, P. Recent developments in regioselective ring opening of aziridines. Tetrahedron 2010, 66, 2549-2560.

8. Chawla, R.; Singh, A.K.; Yadav, L.D.S. Organocatalysis in synthesis and reactions of epoxides and aziridines. $R S C A d v$. 2013, 3, 11385-11403. 
9. Llaveria, J.; Beltran, A.; Díaz-Requejo, M.M.; Matheu, M.I.; Castillon, S.; Perez, P.J. Efficient Silver-Catalyzed Regio- and Stereospecific Aziridination of Dienes. Angew. Chem. Int. Ed. 2010, 49, 7092-7095.

10. Llaveria, J.; Beltran, A.; Sameera, W.M.C.; Locati, A.; Diaz-Requejo, M.M.; Matheu, M.I.; Castillon, S.; Maseras, F.; Perez, P.J. Chemo-, Regio-, and Stereoselective Silver-Catalyzed Aziridination of Dienes: Scope, Mechanistic Studies, and Ring-Opening Reactions. J. Am. Chem. Soc. 2014, 136, 5342-5350.

11. Davis, F.A.; Zhou, P. Asymmetric synthesis of the antibiotic (+)-thiamphenicol using $c i s-N-(p-$ toluenesulfinyl)aziridine 2-carboxylic acids. Tetrahedron 1994, 35, 7525-7528.

12. Davis, F.A.; Reddy, G.V. Aziridine-2-carboxylic acid mediated asymmetric synthesis of D-erythroand L-threo-sphingosine from a common precursor. Tetrahedron Lett. 1996, 37, 4349-4352.

13. Cardillo, G.; Gentilucci, L.; Tolomelli, A.; Tomasini, C. Ring expansion of $N$-acyl aziridine-2-imides to oxazoline-4-imides, useful precursors of pure beta-hydroxy alpha-aminoacids. Tetrahedron Lett. 1997, 39, 6953-6956.

14. Prasad, P.A.B.; Sekar, G.V.; Singh, K. An efficient method for the cleavage of aziridines using hydroxyl compound. Tetrahedron Lett. 2000, 41, 4677-4679.

15. Olofsson, B.; Khamrai, U.; Somfai, P. A regio- and stereodivergent synthesis of vic-amino alcohols. Org. Lett. 2000, 2, 4087-4089.

16. Saha, B.; Nandy, J.P.; Shukla, S.; Siddiqui, I.; Iqbal, J. Stereoselective synthesis of beta-substituted phenylalanine-beta-phenylisoserine-derived tripeptides using $N$-cinnamoyl-L-proline as template: Synthesis of structural analogues of HIV protease inhibitors. J. Org. Chem. 2002, 67, 7858-7860.

17. Prasad, P.A.B.; Sanghi, R.; Singh, K. Studies on ring cleavage of aziridines with hydroxyl compound. Tetrahedron 2002, 58, 7355-7363.

18. Olofsson, B.; Somfai, P. A regio- and stereodivergent route to all isomers of vic-amino alcohols. J. Org. Chem. 2002, 67, 8574-8583.

19. Chakraborty, T.K.; Ghosh, A.; Raju, T.V. Efficient ring opening reactions of $N$-tosyl aziridines with amines and water in presence of catalytic amount of cerium(IV) ammonium nitrate. Chem. Lett. 2003, 32, 82-83.

20. Concellon, J.M.; Riego, E. Ring opening of nonactivated 2-(1-aminoalkyl) aziridines: Unusual regio- and stereoselective C-2 and C-3 cleavage. J. Org. Chem. 2003, 68, 6407-6410.

21. Wang, Z.; Cui, Y.T.; Xu, Z.B.; Qu, J. Hot water-promoted ring-opening of epoxides and aziridines by water and other nucleopliles. J. Org. Chem. 2008, 73, 2270-2274.

22. Li, X.; Ni, B.; Chang, H.-H.; Wei, W.-L. NaHSO 3 -promoted ring openings of $N$-tosylaziridines and epoxides with $\mathrm{H}_{2} \mathrm{O}$. Heterocycles 2014, 89, 1009-1016.

23. Yadav, J.S.; Reddy, B.V.; Sadashiv, K.; Harikishan, K. Indium triflate-catalyzed ring opening of aziridines with carboxylic acid. Tetrahedron Lett. 2002, 43, 2099-2101.

24. Fan, R.-H.; Hou, X.-L. Tributylphosphine-catalyzed ring-opening reaction of epoxides and aziridines with acetic anhydride. Tetrahedron Lett. 2003, 44, 4411-4413.

25. Yadav, J.S.; Reddy, B.V.S.; Sadashiv, K.; Harikishan, K.; Narsaiah, A.V. Acylative cleavage of aziridines with acid anhydrides catalyzed by Scandium triflate. J. Mol. Catal. A 2004, 220, 153-157. 
26. Das, B.; Reddy, V.S.; Tehseen, F. A mild, rapid and highly regioselective ring-opening of epoxides and aziridines with acetic anhydride under solvent-free conditions using ammonium-12molybdophosphate. Tetrahedron Lett. 2006, 47, 6865-6868.

27. Sun, X.; Ye, S.; Wu, J. N-Heterocyclic Carbene: An efficient catalyst for the ring-opening reaction of aziridine with acid anhydride. Eur. J. Org. Chem. 2006, 4787-4790.

28. Li, X.; Li, G.; Chang, H.; Zhang, Y.; Wei, W. Tetrabutylammonium bromide-mediated ring opening reactions of $N$-tosylaziridines with carboxylic acids in DMF. RSC Adv. 2014, 4, 6490-6495.

29. Schroeder, G.; Łeska, B.; Jarczewski, A.; Nowak-Wydra, B.; Brzezinski, B. FTIR, NMR and kinetic studies of proton transfer reactions from nitro-substituted diarylmethanes to $N$-bases with guanidine character. J. Mol. Struct. 1995, 344, 77-88.

30. Coles, M.P. Bicyclic-guanidines, -guanidinates and -guanidinium salts: Wide ranging applications from a simple family of molecules. Chem. Commun. 2009, 3659-3676.

31. Simoni, D.; Rondanin, R.; Morini, M.; Baruchello, R.; Invidata, F.P. 1,5,7-Triazabicyclo [4.4.0]dec-1-ene (TBD), 7-methyl-TBD (MTBD) and the polymer-supported TBD (P-TBD): Three efficient catalysts for the nitroaldol (Henry) reaction and for the addition of dialkyl phosphites to unsaturated systems. Tetrahedron Lett. 2000, 41, 1607-1610.

32. Simoni, D.; Rossi, M.; Rondanin, R.; Mazzali, A.; Baruchello, R.: Malagutti, C.; Roberti, M.; Invidata, F.P. Strong bicyclic guanidine base-promoted Wittig and Horner-Wadsworth-Emmons reactions. Org. Lett. 2000, 2, 3765-3768.

33. Ye, W.; Xu, J. Tan, C.-T.; Tan, C.-H 1,5,7-Triazabicyclo[4.4.0]dec-5-ene (TBD) catalyzed Michael reactions. Tetrahedron Lett. 2005, 46, 6875-6878.

34. Pratt, R.C.; Lohmeijer, B.G.G.; Long, D.A.; Waymouth, R.A.; Hedric, J.A. Triazabicyclodecene: A simple bifunctional organocatalyst for acyl transfer and ring-opening polymerization of cyclic esters. J. Am. Chem. Soc. 2006, 129, 4556-4557.

35. Jiang, Z.; Zhang, Y.; Ye, W.; Tan, C.-H. P-C Bond formation via direct and three-component conjugate addition catalyzed by 1,5,7-triazabicyclo[4.4.0]dec-5-ene (TBD). Tetrahedron Lett. 2007, $48,51-54$.

36. Sabot, C.; Kumar, K.A.; Meunier, S.; Mioskowski, C. A convenient aminolysis of esters catalyzed by 1,5,7-triazabicyclo[4.4.0]dec-5-ene (TBD) under solvent-free conditions. Tetrahedron Lett. 2007, 48, 3863-3866.

37. Sabot, C.; Kumar, K.A.; Antheaume, C.; Mioskowski, C. Triazabicyclodecene: An Effective Isotope Exchange Catalyst in $\mathrm{CDCl}_{3}$. J. Org. Chem. 2007, 72, 5001-5004.

38. Ghobril, C. Sabot, C. Mioskowski, C. Baati, R. TBD-Catalyzed Direct 5- and 6-enolexo Aldolization of Ketoaldehydes. Eur. J. Org. Chem. 2008, 4104-4108.

39. Mahe, O.; Frath, D.; Dez, I.; Marsais, F.; Levacher, V.; Briere, J.-F. TBD-organocatalysed synthesis of pyrazolines. Org. Biomol. Chem. 2009, 7, 3648-3651.

40. Saliu, F.; Rindone, B. Organocatalyzed synthesis of ureas from amines and ethylene carbonate. Tetrahedron Lett. 2010, 51, 6301-6304.

41. Poladura, B.; Martínez-Castañeda, Á.; Rodríguez-Solla, H.; Concellón, C.; del Amo, V. TBD-catalyzed $\alpha$-sulfenylation of cyclic ketones: Desymmetrization of 4-substituted cyclohexanone. Tetrahedron 2012, 68, 6438-6446. 
42. Lanari, D.; Rosati, O.; Curini, M. A solvent-free protocol for the synthesis of 3-formyl-2H-chromenes via domino oxa Michael/aldol reaction. Tetrahedron Lett. 2014, 55, 1752-1755.

43. Deredas, D.; Huben, K.; Maniukiewicz, W.; Krawczyk, H. Highly syn-diastereoselective Michael addition of enolizable ketones to 3-(diethoxyphosphoryl)coumarin. Tetrahedron 2014, 70, 8925-8929.

44. Simón, L.; Goodman, J.M. The Mechanism of TBD-catalyzed ring-opening polymerization of cyclic esters. J. Org. Chem. 2007, 72, 9656-9662.

45. Kiesewetter, M.K.; Scholten, M.; Kirn, N.; Weber, R.L.; Hedrick, J.L.; Waymouth, R.M. Cyclic guanidine organic catalysts: What is magic about triazabicyclodecene? J. Org. Chem. 2009, 74, 9490-9496.

46. Hammar, P.; Ghobril, C.; Antheaume, C.; Wagner, A.; Baati, R.; Himo, F. Theoretical Mechanistic Study of the TBD-Catalyzed Intramolecular aldol reaction of ketoaldehydes. J. Org. Chem. 2010, $75,4728-4736$.

47. Fu, X.; Tan, C.-H. Mechanistic considerations of guanidine-catalyzed reactions. Chem. Commun. 2011, 47, 8210-8222.

48. Matsukawa, S.; Harada, T.; Takahashi, H. TBD-catalyzed ring opening of aziridines with silylated nucleophiles. Synth. Commun. 2013, 43, 406-414.

49. Matsukawa, S.; Takahashi, H.; Takahashi, S. TBD-catalyzed trifluoromethylation of carbonyl compounds with (trifluoromethyl)trimethylsilane. Synth Commun. 2013, 43, 1523-1529.

50. Tomoi, M.; Kato, Y.; Kakiuchi, H. Polymer-supported bases, 2. Polystyrene-supported 1,8-diazabicyclo[5.4.0] undec-7-ene as reagent in organic syntheses. Makromol. Chem. 1984, 185, 2117-2124.

51. Iijima, K.; Fukuda, W.; Tomoi, M. Polymer-supported bases. XI. Esterification and alkylation in the presence of polymer-supported bicyclic amidine or guanidine moieties. J. Macromol. Sci. A Pure Appl. Chem. 1992, 29, 249-261.

52. Xu, W.; Mohan, R.; Morrissey, M.M. Polymer supported bases in combinatorial chemistry: Synthesis of aryl ethers from phenols and alkyl halides and aryl halides. Tetrahedron. Lett. 1997, $38,7337-7340$.

53. Boisnard, S.; Chastanet, J.; Zhu, J. A high throughput synthesis of aryl triflate and aryl nonaflate promoted by a polymer supported base (PTBD). Tetrahedron Lett. 1999, 40, 7469-7472.

54. McNamara, C.A.; Dixon, M.J.; Bradley, M. Recoverable catalysts and reagents using recyclable polystyrene-based supports. Chem. Rev. 2002, 102, 3275-3300.

55. Benaglia, M.; Puglisi, A.; Cozzi, F. Polymer-supported organic catalysts. Chem. Rev. 2003, 103, 3401-3429.

56. Cozzi, F. Immobilization of organic catalysts: When, why, and how. Adv. Synth. Catal. 2006, 348. 1367-1390.

57. Ikegami, S.; Hamamoto, H. Novel recycling system for organic synthesis via designer polymer-gel catalysts. Chem. Rev. 2009, 109, 583-593.

58. Lu, J.; Toy, P.H. Organic polymer supports for synthesis and for reagent and catalyst immobilization. Chem. Rev. 2009, 109, 815-838.

59. Kristensen, T.E.; Hansen, T. Polymer-supported chiral organocatalysts: Synthetic strategies for the road towards affordable polymeric immobilization. Eur. J. Org. Chem. 2010, 17, 3179-3204. 
60. Fringuelli, F.; Pizzo, F.; Vittoriani, C.; Vaccaro, L. Polystyryl-supported TBD as an efficient and reusable catalyst under solvent-free conditions. Chem. Commun. 2004, 2756-2757.

61. Fringuelli, F.; Pizzo, F.; Vittoriani, C.; Vaccaro, L. Polystyrene-supported 1,5,7-triazabicyclo[4.4.0] dec-5-ene as an efficient and reusable catalyst for the thiolysis of 1,2-epoxides under solvent-free conditions. Eur. J. Org. Chem. 2006, 1231-1236.

62. Lanari, D.; Balini, R.; Palmieri, A.; Pizzo, F.; Vaccaro, L. Diastereoselective three-step route to $o$-(6-nitrocyclohex-3-en-1-yl)phenol and tetrahydro-6H-benzo[c]chromen-6-ol derivatives from salicylaldehydes. Eur. J. Org. Chem. 2011, 2874-2884.

63. Matsukawa, S.; Fujikawa, S. Polystyrene-supported TBD as an efficient and reusable organocatalyst for cyanosilylation of aldehydes, ketones, and imines. Tetrahedron Lett. 2012, 53, 1075-1077.

64. Matsukawa, S.; Harada, T.; Yasuda, S. Polystyrene-supported TBD catalyzed ring-opening of $N$-tosylaziridines with silylated nucleophiles. Org. Biomol. Chem. 2012, 10, 4886-4890.

65. Matsukawa, S.; Tsukamoto, K.; Harada, T.; Yasuda, S. An efficient method for opening $N$-tosyl aziridines with silylated nucleophiles using polystyrene-supported TBD as a reusable organocatalyst. Synthesis 2013, 45, 2959-2965.

66. Thakur, V.V.; Sudalai, A. $N$-Bromoamides as versatile catalysts for aziridination of olefins using chloramine-T. Tetrahedron Lett. 2003, 44, 989-992.

Sample Availability: Samples of the compounds $\mathbf{2} \mathbf{a}-\mathbf{2} \mathbf{f}, \mathbf{2} \mathbf{a}^{\prime}-\mathbf{2} \mathbf{f}^{\prime}, \mathbf{2} \mathbf{a}^{\prime \prime}, \mathbf{2} \mathbf{c}^{\prime \prime}$ are available from the authors.

(C) 2015 by the authors; licensee MDPI, Basel, Switzerland. This article is an open access article distributed under the terms and conditions of the Creative Commons Attribution license (http://creativecommons.org/licenses/by/4.0/). 